Chirurg 2019 90 (Suppl 2):S20 https://doi.org/10.1007/s00104-019-0882-8 Online publiziert: 13. Februar 2019 (c) Springer Medizin Verlag GmbH, ein Teil von Springer Nature 2019

\section{Originalpublikation}

Schlottmann F, Luckett DJ, Fine J, Shaheen NJ, Patti MG (2018) Laparoscopic Heller myotomy versus peroral endoscopic myotomy (POEM) for achalasia. A systematic review and metaanalysis. Ann Surg 267:451-460

Hintergrund und Fragestellung. Durch zunehmende Verbreitung der minimalinvasiven Techniken wurde in den letzten zwei Dekaden überwiegend die laparoskopische Heller-Myotomie (LHM) mit partieller Fundoplikation zur symptomatischen Behandlung der Achalasie eingesetzt. Seit Erstbeschreibung der peroralen endoskopischen Myotomie (POEM) im Jahr 2010 hat dieses Verfahren in einigen Zentren die LHM ersetzt, auch wenn randomisierte Studien zu kurz- und langfristigen Ergebnissen fehlen. Wesentlicher Unterschied dieser beiden Techniken ist, dass die POEM im Gegensatz zur LHM nicht mit einer Antirefluxmaßnahme kombiniert wird.

Methoden. Von einer amerikanischen Arbeitsgruppe wurde jetzt die vorliegende Evidenz zu diesem Thema in einer Metaanalyse zusammengefasst, Outcomeparameter der Analyse waren die postoperative Dysphagie und der gastroösophageale Reflux (GERD). 53 Studien mit insgesamt 5834 Patienten untersuchten die LHM, 21 Studien mit 1958 Patienten analysierten das endoskopische Verfahren. Das mediane Followup war signifikant länger für die Pati-

W. Schröder · C. J. Bruns

Klinik für Allgemein-, Viszeral- und Tumorchirurgie, Universitätsklinik Köln, Köln, Deutschland

\title{
Therapie der Achalasie
}

\section{Laparoskopische Heller-Myotomie oder perorale endoskopische Myotomie?}

enten nach LHM (41,4 vs. 16,2 Monate, $p<0,0001)$.

Ergebnisse. Die Wahrscheinlichkeit einer Verbesserung der Dysphagie betrug mit der POEM nach 12 Monaten 93,5\% und nach 24 Monaten $92,7 \%$ und war damit signifikant besser als mit der LHM (12 Monate: 91,0\%, 24 Monate: 90,0\%; für beide $p=0,01)$. Auf der anderen Seite war bei Patienten mit POEM das Risiko größer, postoperativ klinische Symptome einer GERD zu entwickeln (OR 1,69, $95 \%$-KI 1,33-2,14, $p<0,0001)$ und in der Kontrollendoskopie eine erosive Ösophagitis aufzuweisen (OR 9,31, 95\%-KI $4,71-18,85, p<0,0001)$. GERD mit einer pathologischen $\mathrm{pH}$-Messung wurde bei $11,1 \%$ der Patienten nach LHM und $47,5 \%$ der Patienten nach POEM beobachtet.

Fazit. Die vorliegenden Daten der Metaanalyse zeigen, dass POEM im Vergleich zur LHM zwar zu einer besseren Schluckfunktion postoperativ führt, dass dieser Vorteil aber mit einer ausgeprägten gastroösophagealen Refluxerkrankung erkauft wird. Diese Komplikation wird durch Kombination der LHM mit einer Antirefluxoperation weitestgehend vermieden. Die in dieser Form zum ersten Mal publizierten Ergebnisse sollten in einem Behandlungsalgorithmus der Achalasie differenziert nach dem Alter der Patienten Berücksichtigung finden.

\section{Korrespondenzadresse}

Prof. Dr. W. Schröder, FACS, FEBS

Klinik für Allgemein-, Viszeral- und

Tumorchirurgie, Universitätsklinik Köln Kerpener Str. 62, 50937 Köln, Deutschland wolfgang.schroeder@uk-koeln.de

Interessenkonflikt. W. Schröder und C. J. Bruns geben an, dass kein Interessenkonflikt besteht. 